\title{
Clinical and molecular characteristics of 69 Chinese patients with ornithine transcarbamylase deficiency
}

Deyun Lu' ${ }^{1 \dagger}$, Feng Han ${ }^{2 \dagger}$, Wenjuan Qiu' ${ }^{1}$, Huiwen Zhang ${ }^{1}$, Jun Ye ${ }^{1}$, Lili Liang ${ }^{1}$, Yu Wang ${ }^{1}$, Wenjun Ji ${ }^{1}$, Xia Zhan ${ }^{1}$, Xuefan $\mathrm{Gu}^{1}$ and Lianshu Han ${ }^{1 *}$

\begin{abstract}
Background: This study aimed to describe the clinical and biochemical features of Chinese patients with ornithine transcarbamylase deficiency (OTCD), and to investigate the mutation spectrum of OTC gene and their potential correlation with phenotype.
\end{abstract}

Methods: Sixty-nine patients with OTCD were enrolled between 2004 and 2019. Clinical and laboratory data were reviewed retrospectively from medical records.

Results: Fifteen cases (13 males, 2 females) presented with early onset; 53 cases ( 21 males, 32 females) had late onset, and one female was asymptomatic. The median onset age was 1.5 years (range 1 day-56 years). Urine orotic acid levels were increased in all patients tested, while only $47.6 \%$ of patients showed decreased serum levels of citrulline. The peak plasma ammonia levels were higher in early-onset patients than in late-onset patients $(P<0.01)$. Fifty-four different mutations of OTC gene were identified and 18 of them were novel. R277W (10.6\%) was the most common mutation, followed by G195R (4.6\%) and A209V (3.0\%). By June 2019, 41 patients had survived, 24 were deceased, and 4 were lost to follow-up. Among the survivors, 13 patients had received liver transplantation at a median age of 3 years, with a one-year survival rate of 100\%. The mortality of OTCD is extremely high among patients with early onset ( $80.0 \%$ versus $24.5 \%$ in patients with late onset).

Conclusions: The evaluation of serum citrulline level is of limited value in diagnosis of OTCD, while urine orotic acid detection and genetic testing are more helpful.

Keywords: Hyperammonemia, Urea cycle disorders, Ornithine transcarbamylase deficiency, OTC, Gene mutation

\section{Introduction}

Ornithine transcarbamylase deficiency (OTCD, MIM \#300461) is one of the most common urea cycle disorders and accounts for approximately half of the inherited urea cycle disorders. The estimated prevalence

\footnotetext{
*Correspondence: hanlianshu@xinhuamed.com.cn

tDeyun Lu and Feng Han contributed equally to this work

${ }^{1}$ Department of Pediatric Endocrinology and Genetic Metabolism,

Shanghai Institute for Pediatric Research, Xinhua Hospital Affiliated To

Shanghai Jiao Tong University School of Medicine, Shanghai, China

Full list of author information is available at the end of the article
}

is $1 / 14,000-1 / 77,000$ [1]. Ornithine transcarbamylase catalyzes the formation of citrulline from ornithine and carbamyl phosphate.[2]. Thereby, OTCD leads to excess carbamoyl phosphate production, which then reacts with aspartate generating excess orotic acid. The human OTC gene is located on Xp21.1 with a full-length of $73 \mathrm{~kb}$ containing 10 exons and 9 introns [3]. Mutations of the OTC gene are highly heterogeneous and the recurrent sequence variants varied by ethnic background. The most common mutations are $\mathrm{R} 40 \mathrm{H}$ and $\mathrm{R} 227 \mathrm{~W}$ in Japan [4], R129H, R40H, and G195R in Spain [5, 6], but yet unclear in China.

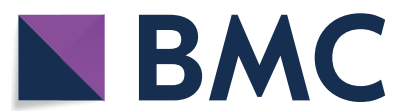

(c) The Author(s) 2020. Open Access This article is licensed under a Creative Commons Attribution 4.0 International License, which permits use, sharing, adaptation, distribution and reproduction in any medium or format, as long as you give appropriate credit to the original author(s) and the source, provide a link to the Creative Commons licence, and indicate if changes were made. The images or other third party material in this article are included in the article's Creative Commons licence, unless indicated otherwise in a credit line to the material. If material is not included in the article's Creative Commons licence and your intended use is not permitted by statutory regulation or exceeds the permitted use, you will need to obtain permission directly from the copyright holder. To view a copy of this licence, visit http://creativecommons.org/licenses/by/4.0/. The Creative Commons Public Domain Dedication waiver (http://creativeco mmons.org/publicdomain/zero/1.0/) applies to the data made available in this article, unless otherwise stated in a credit line to the data. 
OTCD is an X-linked recessive genetic disorder. Therefore, almost all hemizygous males develop this disease. Approximately $20 \%$ of heterozygous females present some neurocognitive disorders due to unfavorable random X-inactivation. However, the clinical picture of carrier females is highly diverse because of the extent of X-inactivation in hepatocytes [7]. The OTCD clinical phenotypes can generally be divided into two groups, namely early-onset (onset age $\leq 30$ days), and late-onset (onset age $>30$ days) or asymptomatic. The major symptoms include vomiting, apastia, lethargy, convulsion, muscle hypotonia, and coma. These symptoms can be precipitated or aggravated by high protein meals, fasting, infections, trauma, surgery, or childbirth [8]. The survival rate is $70-90 \%$ among female patients, and $50-60 \%$ among male patients [9-11].

Here, we report on the clinical and biochemical features, as well as molecular characteristics of 69 unrelated Chinese OTCD patients. As such, we aimed to summarize the mutation spectrum of the OTC gene in Chinese patients and analyze the relationship between phenotype and genotype.

\section{Materials and methods Patients}

In this study, 69 unrelated patients (34 males and 35 females) diagnosed with OTCD were recruited between 2004 and 2019. The diagnosis of OTCD was based on clinical features, specific biochemical criteria (high blood ammonia, high plasma glutamine, low plasma citrulline, and high urinary excretion of orotic acid), as well as molecular analysis. Clinical manifestations and courses, as well as biochemical data were collected and reviewed retrospectively from medical records.

\section{Biochemical detection}

The quantification of blood amino acids was tested by tandem mass spectrometry (MS/MS; Applied Biosystems, API 4000, California, United State) on dried blood spots [12]. The levels of urinary organic acids were measured by gas chromatography -mass spectrometry (GCMS; Shimadzu Limited, QP2010, Kyoto, Japan) [13].

\section{Molecular analysis of the OTC gene}

After obtaining informed consent, $2 \mathrm{ml}$ of peripheral blood was drawn from the patients and their parents. Samples from 66 unrelated patients were used for OTC molecular tests. The other 3 patients (P11-13) could not be included in the analysis as they died of acute hyperammonemic encephalopathy shortly after birth, so samples from their mothers were used for genetic test. Genomic DNA was extracted from peripheral blood leukocytes by using a blood kit (Zeesan Biotech, China). Polymerase chain reaction (PCR) was used to amplify all coding exons and exon-intron boundaries of the OTC gene using 9 pairs of synthetic oligonucleotide primers, and the primer sequences and annealing temperature were showed in Additional file 1: Table 1S. The PCR products were then purified and bidirectionally sequenced using the Applied Biosystems 96-capillary 3730XL system. Three patients underwent whole-exome sequencing (WES). The library was sequenced using Illumina HiSeq 4000 and generated 150 bp paired-end reads. Data analysis was performed as previously described [14]. Multiplex ligation-dependent probe amplification analysis (MLPA; SALSA MLPA P079 OTC kit, MRC-Holland, Amsterdam, The Netherlands) was performed for samples in which Sanger sequencing or WES failed to detect any disease-causing mutation $[15,16]$.

\section{Bioinformatics analysis}

The variants were called novel if they were not listed in the Human Gene Mutation Database, ClinVarMiner database, and Exome Aggregation Consortium database. For the novel missense variants, potential pathogenicity was analyzed using PolyPhen-2, PROVEAN, Mutation Taster, Sorting Intolerant From Tolerant (SIFT), and Amino acid conservation was analyzed by phyloP and phastCons.

\section{Statistical methods}

Data were expressed as the median (range, minimum and maximum) for quantitative variables and percentages for qualitative variables. The level of statistical significance was obtained using the Mann-Whitney $U$ test for quantitative variables.

\section{Results}

\section{Diagnostic mode}

In this study, 15 patients underwent the newborn screening by MS/MS using blood citrulline as a biomarker. However, only 4 cases (P1, P7, P15, and P35) were identified, and 3 of them (P1, P7, and P35) with early onset phenotype showed first disease onset before the newborn screening results were available, so only one case (P15) was diagnosed through the newborn screening before the onset of symptoms. Of the other 11 patients missed by newborn screening, 9 had late onset of disease, one (P9) had severe early onset, and one (P43) was asymptomatic. Therefore, most $(64 / 69,92.8 \%)$ of patients were diagnosed by selective metabolic investigation after the onset of symptoms, whereas identification by newborn screening $(4 / 69,5.8 \%)$ or prenatal testing $(1 / 69,1.4 \%)$ was less frequent. 


\section{Clinical presentations}

With the exception of one asymptomatic female patient, the age at onset of 68 patients was shown in Fig. 1. Fifteen cases (21.7\%), 13 males and 2 females, suffered first hyperammonemic episode during neonatal period, and the median onset age was 3 days (range 1-27 days). The remaining 53 cases (76.8\%), 21 males and 32 females, had late-onset OTCD, and the median onset age was 2 years (range 3 months-56 years). Over all, three quarters of patients underwent the first hyperammonemic episode within 3 years of age, and the peak age was neonatal period in males and 1-2 years in females, respectively.

Clinical symptoms of these patients were summarized in Fig. 2. Neurological symptoms were the most common clinical manifestations in both early-onset and lateonset groups. The acute severe neurological symptoms (including coma, lethargy and seizures) occurred in 14 cases $(93.3 \%)$ in the early-onset and 33 cases $(62.3 \%)$ in the late-onset group, indicating clinical symptoms were more serious in early-onset group. The gastrointestinal symptoms ranked second in both groups; vomiting was the most prominent symptom.

\section{Biochemical results at OTCD diagnosis}

The results of the biochemical studies at diagnosis were showed in Table 1 . The urinary orotic acid was significantly elevated in most of tested patients (53/56) and slightly elevated in three patients (P28, P34, and P69), whereas decreased serum citrulline was found in only $47.6 \%(30 / 63)$ of patients. The elevated levels of blood ammonia were found in all patients tested, serum glutamine in $22.2 \%(12 / 54)$ of patients, and uracil in $94.4 \%$
$(51 / 54)$ of patients, respectively. In addition, $73.1 \%$ $(38 / 52)$ of patients had elevated plasma alanine aminotransferase (ALT), among whom 73.7\% (28/38) showed severe elevation of ALT. International normalized ratio was elevated in 11 patients tested.

We also compared the biochemical data between male and female patients (Table 2). Among the patients with detected citrulline concentrations, $69.0 \%(20 / 29)$ of male patients and $29.4 \%(10 / 34)$ of female patients had decreased serum citrulline concentrations, with significantly lower levels of serum citrulline concentrations in the male group $(P<0.01)$. However, there were no significant differences in other metabolic characteristics between male and female groups $(P>0.05)$.

In addition, the biochemical data were compared between early-onset and late-onset groups (Table 2). The peak ammonia levels in the early-onset group were much higher than those in the late-onset group $(P<0.01)$, which indicated that it may be associated with disease severity. Of all patients, $91.7 \%$ (11/12) with early-onset disease and $37.3 \%(19 / 51)$ with late-onset disease showed decreased citrulline concentrations. The citrulline concentration was significantly lower in patients with earlyonset $(P<0.01)$. Nevertheless, blood glutamine, urine orotic acid and uracil concentration were similar between the two groups $(P>0.05)$.

\section{Mutation analysis of the OTC gene}

We identified 54 different mutations in 63 unrelated patients including 28 (51.9\%) missense mutations, 5 (9.3\%) nonsense mutations, 8 (14.8\%) splicing mutations, 7 (13.0\%) gross deletions, 3 (5.6\%) small deletions,

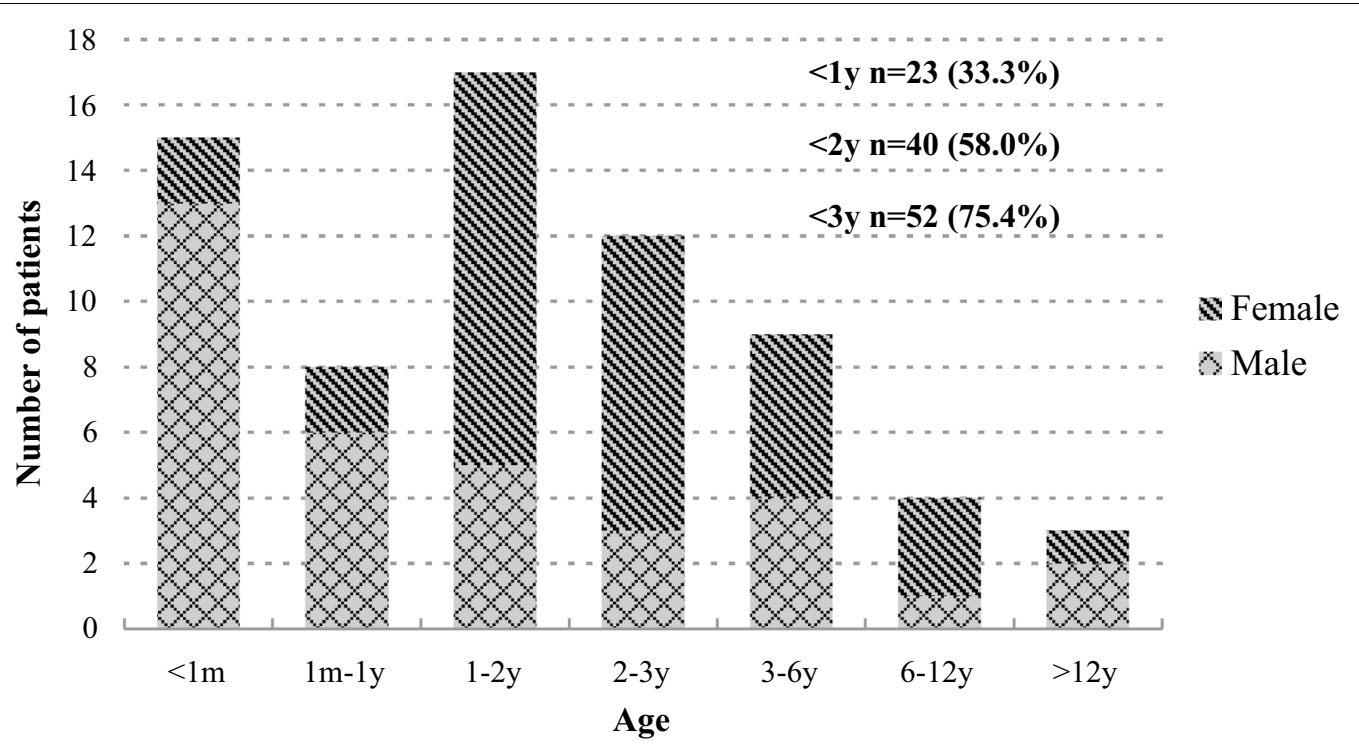

Fig. 1 Age at onset of 68 Chinese patients with OTCD 


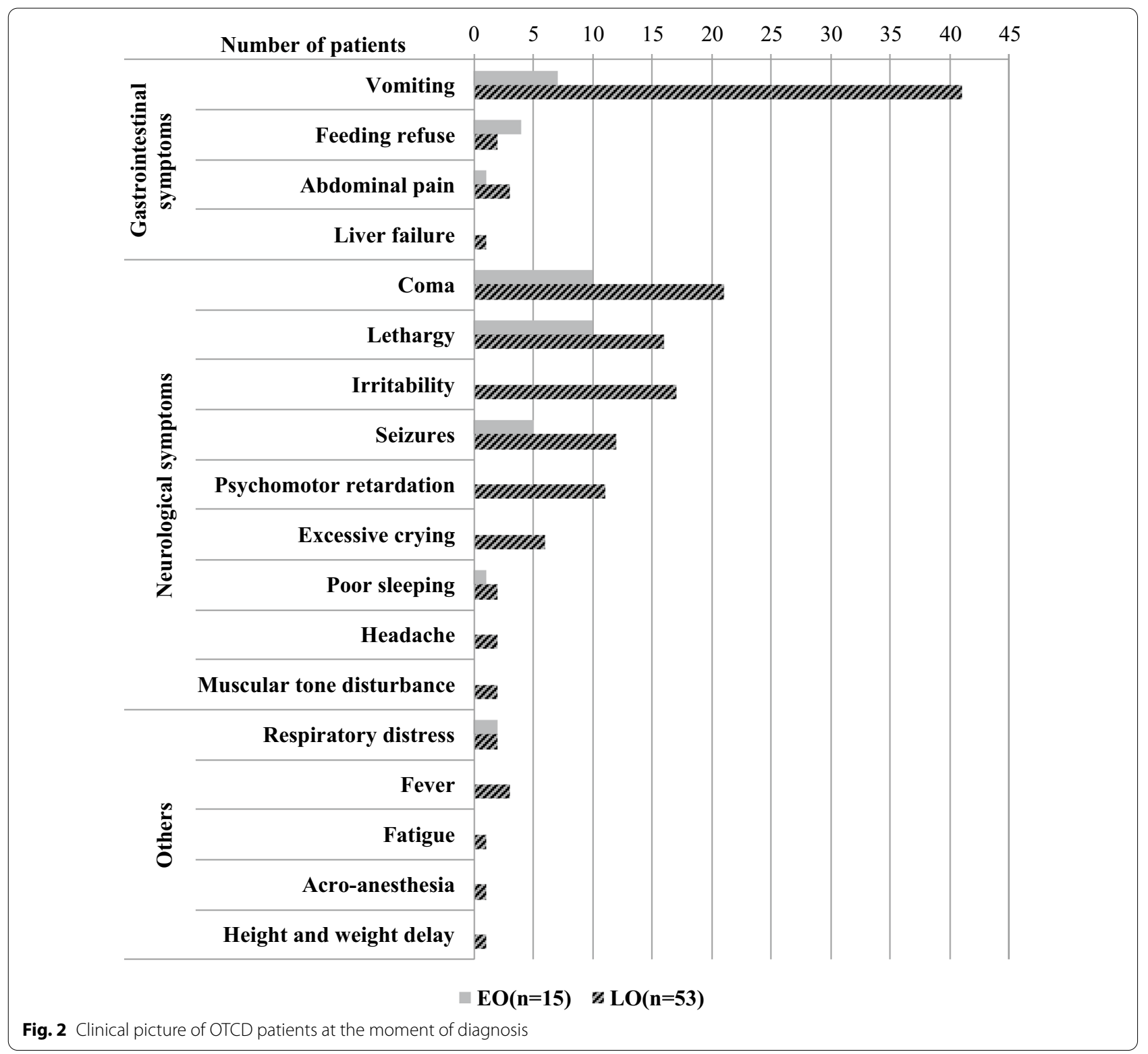

2 (3.7\%) gross duplications, and 1(1.9\%) small insertion (Table 3). In 3 patients (P67-P69), the results of WES were negative, but no MPLA was performed due to parental refusal. De novo mutations had been found in $36.2 \%(25 / 69)$ of the patients in this study. Mutations observed in male patients were mainly distributed in exon 6, 8, and 9, while mutations observed in females were highly dispersed. The most common mutations in our cohort were R277W (7/66, 10.6\%), followed by G195R (3/66; 4.6\%) and A209V (2/66; $3.0 \%)$. Of all mutations, 18 were novel: $\mathrm{c} .77+1 \mathrm{G}>\mathrm{C}$ $(\mathrm{IVS} 1+1 \mathrm{G}>\mathrm{C}), \quad$ c.78-2 A $>\mathrm{G} \quad($ IVS1-2A $>\mathrm{G}), \quad$ c.122A $>\mathrm{C}$ (p.D41A), c.124_126delCTT (p.42delL), c.207-226del20,
c.552insGAAC (p.S185Efs*41), c.703C >T (p.Q235X), c.704A>C (p.Q235P), c.860C >T (p.T287I), c.868-1 G>C (IVS8-1G>C), c.913C >T(p.P305S), E1-4 deletion, E1-4 duplication, E2-6 duplication, E5-8 deletion, E7-10 deletion, E9-10 deletion, and 7.8 Mb deletion of Xp11.4p21.2, which have not been previously reported in the literature or registered in the HGMD, ClinVarMiner, ExAC and gnomAD database.

The bioinformatic characteristics of the 4 novel missense variants (D41A, Q235P, T287I, and P305S) are shown in Table 4. D41 interlinks with carbamoyl phosphate domain through hydrogen bonds. The known mutant D41G has been predicted to decrease the stability 
Table 1 Clinical biochemical findings at presentation in 69 patients with OTCD

\begin{tabular}{|c|c|c|c|c|c|c|c|c|c|c|}
\hline Patient No & Sex & Onset age & $\begin{array}{l}\text { Current/ } \\
\text { death age }\end{array}$ & Type & $\begin{array}{l}\mathrm{CIT}^{\mathrm{a}} \\
(\mu \mathrm{mol} / \mathrm{L})\end{array}$ & $\begin{array}{l}\mathrm{GLN}^{\mathrm{a}} \\
(\mu \mathrm{mol} / \mathrm{L})\end{array}$ & $\begin{array}{l}\text { Orotic acid } \\
\text { (mmol/molCr) }\end{array}$ & $\begin{array}{l}\text { Uracil }^{\mathrm{a}} \\
(\mathrm{mmol} / \mathrm{molCr})\end{array}$ & $\begin{array}{l}\text { Peak AMON } \\
(\mu \mathrm{mol} / \mathrm{L})\end{array}$ & $\begin{array}{l}\text { ALT }^{\mathrm{a}} \\
\text { (U/L) }\end{array}$ \\
\hline $1^{b}$ & M & $7 d$ & $8 m$ & EO, NBS & 1.71 & 3.25 & 425.42 & 8.60 & 300 & 32 \\
\hline 2 & M & $3 d$ & $7 d$ & EO & ND & ND & ND & ND & 346 & 18 \\
\hline 3 & M & $20 d$ & $24 d$ & EO & ND & ND & ND & ND & ND & ND \\
\hline 4 & M & $3 d$ & $7 d$ & EO & 3.09 & 33.54 & 231.20 & 151.20 & 637 & ND \\
\hline 5 & M & $9 d$ & $44 d$ & EO & 4.05 & ND & ND & ND & 815 & ND \\
\hline 6 & M & $3 d$ & $7 d$ & EO & 2.48 & 80.60 & 267.60 & 53.26 & 700 & 18 \\
\hline $7^{b}$ & M & $5 d$ & $3.1 y$ & EO & 3.24 & 15.15 & 22.28 & 8.70 & 50 & 172 \\
\hline 8 & M & $1 d$ & $5 d$ & $\mathrm{EO}$ & 2.18 & 24.79 & 127.33 & 90.78 & $>500$ & 15 \\
\hline $9^{b}$ & M & $3 d$ & $7 d$ & EO,NBS & 4.80 & 43.73 & 1638.32 & 61.91 & 780 & ND \\
\hline 10 & M & $3 d$ & $7 d$ & EO & 4.96 & ND & 214.77 & 9.89 & 1015 & ND \\
\hline 11 & M & $2 d$ & $6 d$ & EO & 3.25 & 45.78 & 423.71 & 256.54 & $>500$ & ND \\
\hline 12 & M & $2 d$ & $2 d$ & EO & 3.40 & ND & ND & ND & 657 & 648 \\
\hline 13 & M & $1 d$ & $7 d$ & EO & ND & ND & ND & ND & 1340 & ND \\
\hline 14 & M & $56 y$ & $56 y$ & LO & 8.83 & 5.84 & 827.28 & 28.69 & 1152 & 23 \\
\hline $15^{b}$ & M & $1.8 y$ & $2.3 y$ & LO,NBS & 3.07 & 27.20 & ND & 17.93 & 180 & 42 \\
\hline 16 & M & $8 m$ & $2.5 y$ & LO & ND & $\mathrm{ND}$ & ND & ND & 300 & ND \\
\hline 17 & $M$ & $2 y$ & $*$ & LO & 5.74 & 10.83 & 65.68 & 29.17 & 218 & ND \\
\hline $18^{b}$ & M & $4 m$ & $9.7 y$ & LO & 6.83 & 44.39 & 228.79 & 311.67 & 350 & 28 \\
\hline 19 & $M$ & $36 y$ & $39 y$ & LO & 3.87 & 17.93 & ND & ND & 427 & 36 \\
\hline 20 & M & $4.3 y$ & $4.3 y$ & LO & 21.74 & 16.37 & 312.35 & 248.40 & 114 & 244 \\
\hline $21^{b}$ & $M$ & $1.4 y$ & $4.7 y$ & LO & 5.62 & 9.92 & 413.21 & 143.06 & 351 & 55 \\
\hline $22^{b}$ & M & $2.6 y$ & $4.4 y$ & LO & ND & ND & ND & ND & 244 & ND \\
\hline $23^{b}$ & M & $1.4 y$ & $2.4 y$ & LO & 25.43 & 5.61 & 137.00 & 6.07 & 114 & 40 \\
\hline 24 & M & $9.7 y$ & $9.9 y$ & LO & 8.58 & 11.23 & 96.00 & 42.90 & 120 & 69 \\
\hline 25 & M & $10 \mathrm{~m}$ & $1.4 y$ & LO & 2.28 & ND & ND & ND & 131 & 327 \\
\hline 26 & M & $3 y$ & $10.8 y$ & LO & 7.48 & 22.23 & 48.31 & 165.31 & 289 & 119 \\
\hline 27 & $M$ & $7 \mathrm{~m}$ & $2.2 y$ & LO & 1.10 & 14.28 & 543.54 & 130.02 & 392 & 462 \\
\hline 28 & M & $1 y$ & $8.7 y$ & LO & 7.09 & 14.21 & 1.85 & 19.79 & 45 & 26 \\
\hline 29 & $M$ & $1.2 y$ & $8.1 y$ & LO & 3.86 & 15.48 & 455.63 & 133.00 & 187 & 48 \\
\hline 30 & M & $8 m$ & $1.8 y$ & LO & 13.60 & $\mathrm{ND}$ & 82.82 & 339.11 & 335 & 1782 \\
\hline 31 & M & $2.1 y$ & $3 y$ & LO & 12.77 & 13.53 & 11.60 & 17.53 & 257 & 116 \\
\hline 32 & M & $5 y$ & $5 y$ & LO & 2.80 & ND & 172.80 & 27.80 & 2500 & 19.7 \\
\hline $33^{b}$ & M & $3.4 y$ & $4 y$ & LO & 18.62 & 14.04 & 77.30 & 62.90 & 447 & 228.9 \\
\hline 34 & $M$ & $3 \mathrm{~m}$ & $4.5 y$ & LO & 2.43 & 12.81 & 1.79 & 5.72 & 265 & 105 \\
\hline $35^{b}$ & $\mathrm{~F}$ & $1 d$ & $1 \mathrm{~m}$ & EO,NBS & 2.88 & 21.31 & 282.12 & 34.56 & 2000 & ND \\
\hline 36 & $F$ & $27 d$ & $8.8 y$ & EO & 14.83 & 29.73 & 197.00 & 51.80 & 410 & 2602 \\
\hline 37 & $\mathrm{~F}$ & $6 y$ & $9.6 y$ & LO & 19.24 & 14.22 & 198.45 & 205.32 & 194 & 509 \\
\hline 38 & $F$ & $2.5 y$ & $4.5 y$ & LO & 15.75 & 13.50 & 202.00 & 67.50 & 190 & 665 \\
\hline 39 & $\mathrm{~F}$ & $5.6 y$ & $5.6 y$ & LO & 12.60 & 11.65 & 189.20 & 50.04 & 500 & 759 \\
\hline 40 & $\mathrm{~F}$ & $2 y$ & $*$ & LO & 14.07 & 18.96 & 141.39 & 35.73 & ND & ND \\
\hline 41 & F & $1.5 y$ & $2 y$ & LO & 8.81 & 9.87 & 48.92 & 71.86 & 103 & 508 \\
\hline 42 & $\mathrm{~F}$ & $2.3 y$ & $5.1 y$ & LO & 9.70 & 12.78 & 43.50 & 251.00 & 1300 & 1330 \\
\hline $43^{b}$ & $F$ & No & $4 m$ & AS,PT & 6.61, & ND & 224.31 & ND & 59 & 28 \\
\hline 44 & $F$ & $2.3 y$ & $3.8 y$ & LO & 11.84 & 4.93 & 43.83 & 131.88 & 120 & 215 \\
\hline 45 & $\mathrm{~F}$ & $2 y$ & $2.8 y$ & LO & 7.68 & 32.68 & ND & ND & 256 & ND \\
\hline 46 & $\mathrm{~F}$ & $2.4 y$ & $3.9 y$ & LO & 13.44 & 61.66 & 673.28 & 161.53 & 385 & 380 \\
\hline 47 & $F$ & $6 \mathrm{~m}$ & $3.8 y$ & LO & 12.68 & 20.81 & 1435.31 & 153.89 & 387 & 214 \\
\hline 48 & F & $29 y$ & $29 y$ & LO & 8.41 & 18.16 & 38.75 & 51.55 & 421 & ND \\
\hline 49 & $\mathrm{~F}$ & $1.5 y$ & $8.4 y$ & LO & 15.90 & 23.87 & 457.51 & 112.32 & 133 & ND \\
\hline
\end{tabular}


Table 1 (continued)

\begin{tabular}{|c|c|c|c|c|c|c|c|c|c|c|}
\hline Patient No & Sex & Onset age & $\begin{array}{l}\text { Current/ } \\
\text { death age }\end{array}$ & Type & $\begin{array}{l}\mathrm{ClT}^{\mathrm{a}} \\
(\mu \mathrm{mol} / \mathrm{L})\end{array}$ & $\begin{array}{l}\mathrm{GLN}^{\mathrm{a}} \\
(\mu \mathrm{mol} / \mathrm{L})\end{array}$ & $\begin{array}{l}\text { Orotic acid }^{\mathrm{a}} \\
(\mathrm{mmol} / \mathrm{molCr})\end{array}$ & $\begin{array}{l}\text { Uracil }^{\mathrm{a}} \\
(\mathrm{mmol} / \mathrm{molCr})\end{array}$ & $\begin{array}{l}\text { Peak AMON } \\
(\mu \mathrm{mol} / \mathrm{L})\end{array}$ & $\begin{array}{l}\text { ALT }^{\mathrm{a}} \\
(\mathrm{U} / \mathrm{L})\end{array}$ \\
\hline $50^{b}$ & $\mathrm{~F}$ & $2.3 y$ & $2.8 y$ & LO & 15.66 & 37.02 & 251.06 & 42.70 & 300 & ND \\
\hline 51 & $\mathrm{~F}$ & $3 y$ & $13.2 y$ & LO & 10.91 & 31.70 & 356.42 & 65.03 & 286 & 422 \\
\hline $52^{b}$ & $\mathrm{~F}$ & $1.5 y$ & $3.3 y$ & LO & 1.77 & $\mathrm{ND}$ & ND & $\mathrm{ND}$ & 350 & 1455.00 \\
\hline 53 & $\mathrm{~F}$ & $1.1 \mathrm{y}$ & $3.2 y$ & LO & 4.27 & ND & 469.38 & 261.67 & 260 & 72 \\
\hline 54 & F & $1.8 y$ & $9.3 y$ & LO & 5.94 & 15.18 & 204.05 & 83.30 & 216 & 415 \\
\hline $55^{b}$ & $\mathrm{~F}$ & $10 \mathrm{~m}$ & $2.6 y$ & LO & ND & ND & ND & ND & 315 & 885 \\
\hline 56 & $\mathrm{~F}$ & $3.5 y$ & $5.1 y$ & LO & 9.53 & 17.52 & 38.21 & 6.75 & 234 & 687 \\
\hline 57 & $\mathrm{~F}$ & $1.3 y$ & $7 y$ & LO & 8.21 & 26.24 & 227.12 & 441.98 & 189 & 139 \\
\hline 58 & $F$ & $1 y$ & $2.8 y$ & LO & 4.21 & 21.26 & 4.53 & ND & 480 & 97 \\
\hline 59 & F & $6 y$ & $6.7 y$ & LO & 10.40 & 42.54 & 199.30 & ND & 170 & 338.2 \\
\hline 60 & $F$ & $2 y$ & $8.4 y$ & LO & 15.56 & 17.72 & 305.73 & 48.85 & 278 & 964 \\
\hline 61 & F & $4 y$ & $9.9 y$ & LO & 5.69 & 23.48 & 19.93 & 338.64 & $>500$ & 839 \\
\hline 62 & F & $1.1 y$ & $2.6 y$ & LO & 3.17 & 14.11 & 77.65 & 29.97 & 300 & 303 \\
\hline 63 & $F$ & $1.4 y$ & $7.3 y$ & LO & 8.82 & 49.73 & 691.94 & 559.21 & 197 & 1639 \\
\hline 64 & $\mathrm{~F}$ & $1 y$ & $7.3 y$ & LO & 3.70 & 5.18 & 55.46 & 156.57 & 383 & 49 \\
\hline 65 & F & $9.3 y$ & $*$ & LO & 7.44 & 56.51 & 106.88 & 61.87 & 268 & ND \\
\hline $66^{b}$ & F & $1.7 y$ & $3.3 y$ & LO & 11.54 & 20.67 & 129.10 & 107.17 & $>500$ & 623 \\
\hline 67 & F & $5.5 y$ & $5.7 y$ & LO & 7.57 & 19.38 & 308.70 & 78.95 & 231 & 311 \\
\hline 68 & F & $2 y$ & * & LO & 6.94 & 29.11 & 213.13 & 164.51 & 256 & 388 \\
\hline 69 & F & $1.5 y$ & $5.7 y$ & LO & 12.37 & 24.27 & 2.72 & 71.62 & 320 & 111 \\
\hline
\end{tabular}

$\mathrm{M}$, male; $\mathrm{F}$, female; d, days; m, months; y, years; EO, early-onset; LO, late-onset; AS, asymptomatic; NBS, identified by newborn screening; PT, identified by prenatal testing; CIT, blood citrulline; GLN, blood glutamine; AMON, blood ammonia; ND, not detected

"*" donates loss to follow-up

a Normal reference values: CIT 7-35 umol/L; GLN 6-30 umol/L; Urine orotic acid 0-1.5 mmol/molCr; Uracil: 0-7 mmol/molCr; AMON 9-30 $\mu \mathrm{mol} / \mathrm{L} ;$ ALT 9-52U/L; severe elevation of ALT was defined as $>175 \mathrm{U} / \mathrm{L}$ in females, $>200 \mathrm{U} / \mathrm{L}$ in males

b The patients who underwent expanded newborn screening by tandem mass spectrometry

Table 2 Comparison of biochemical data between different gender groups and between different phenotypes

\begin{tabular}{|c|c|c|c|c|c|}
\hline & $\begin{array}{l}\mathrm{CIT} \\
(\mu \mathrm{mol} / \mathrm{L})\end{array}$ & $\begin{array}{l}\mathrm{GLN} \\
(\mu \mathrm{mol} / \mathrm{L})\end{array}$ & $\begin{array}{l}\text { Orotic acid } \\
(\mathrm{mmol} / \mathrm{molCr})\end{array}$ & $\begin{array}{l}\text { Uracil } \\
\text { ( } \mathrm{mmol} / \mathrm{molCr} \text { ) }\end{array}$ & $\begin{array}{l}\text { AMON } \\
(\mu \mathrm{mol} / \mathrm{L})\end{array}$ \\
\hline Reference values & $7-35$ & $6-30$ & $0-1.5$ & $0-7$ & $9-30$ \\
\hline Male $(n=34)$ & $4.1(29)$ & $15.1(23)$ & $193.8(24)$ & $53.3(25)$ & $335(33)$ \\
\hline Range & $1.1-25.4$ & $3.3-80.6$ & $1.8-1638.3$ & $5.7-339.1$ & $45-2500$ \\
\hline Female $(n=34)$ & $9.2(34)$ & $20.8(31)$ & $198.9(32)$ & $79.0(29)$ & $273(34)$ \\
\hline Range & $1.8-19.2$ & $4.9-61.7$ & $2.7-1435.3$ & $6.8-559.2$ & $59-2000$ \\
\hline Z & -3.061 & -1.268 & -0.248 & -1.795 & -1.117 \\
\hline$P$ & 0.002 & 0.205 & 0.804 & 0.073 & 0.264 \\
\hline $\mathrm{EO}(\mathrm{n}=15)$ & $3.3(12)$ & $29.7(9)$ & $249.4(10)$ & $52.5(10)$ & $679(14)$ \\
\hline Range & $1.7-14.8$ & $3.3-80.6$ & $22.3-1638.3$ & $8.6-256.5$ & $50-2000$ \\
\hline $\mathrm{LO}(\mathrm{n}=53)$ & $8.4(51)$ & $17.7(45)$ & $181.0(46)$ & $75.4(44)$ & $265(53)$ \\
\hline Range & $1.1-25.4$ & $4.9-61.7$ & $1.8-1435.3$ & $5.7-559.2$ & $45-2500$ \\
\hline Z & -3.326 & -1.938 & -1.455 & -1.336 & -3.726 \\
\hline$P$ & 0.001 & 0.053 & 0.146 & 0.182 & 0.000 \\
\hline
\end{tabular}

Quantitative data were expressed as the median (number of patients tested) and the range (minimum-maximum)

EO, early onset patients; LO, late onset patients 
Table 3 Molecular detective results, family history, and clinical outcomes identified in 69 patients with OTCD

\begin{tabular}{|c|c|c|c|c|c|c|c|c|}
\hline NO. $^{a}$ & Type & Exon/Intron & Nucleotide change & Amino acid change & Nature of mutation & Family history & Therapy received ${ }^{\mathbf{b}}$ & $\begin{array}{l}\text { Outcome/ } \\
\text { neurologic } \\
\text { damage }\end{array}$ \\
\hline 1 & EO & E4 & c. $386 \mathrm{G}>\mathrm{A}$ & p.R129H & NA & No & LPD, drug & Alive/no \\
\hline 2 & EO & E5 & c. $482 A>G$ & p.N161S & Inherited & Yes & ND & Deceased \\
\hline 3 & EO & E6 & c. $579 \mathrm{G}>\mathrm{A}$ & p.W193X & Inherited & Yes & ND & Deceased \\
\hline 4 & EO & E6 & c.583G $>A$ & p.G195R & Inherited & Yes & ND & Deceased \\
\hline 5 & EO & E8 & c. $725 C>T$ & p.T2421 & Inherited & No & LPD, drug & Deceased \\
\hline 6 & EO & E8 & c. $829 C>T$ & p.R277W & De novo & No & $M V, T P N, P D$ & Deceased \\
\hline 7 & $\mathrm{EO}$ & E8 & $c .860 C>T$ & p.T287>l & Inherited & Yes & Drug & Alive/no \\
\hline 8 & $\mathrm{EO}$ & E9 & c. $904 C>T$ & p.H302Y & Inherited & No & Drug & Deceased \\
\hline 9 & $\mathrm{EO}$ & E2-6 & E2-6 duplication & & Inherited & No & ND & Deceased \\
\hline 10 & $\mathrm{EO}$ & E7-10 & E7-10 deletion & & Inherited & Yes & ND & Deceased \\
\hline 11 & $\mathrm{EO}$ & NA & NA & NA & NA & No & ND & Deceased \\
\hline 12 & EO & NA & NA & NA & NA & No & ND & Deceased \\
\hline 13 & EO & NA & NA & NA & NA & No & Drug & Deceased \\
\hline 14 & LO & E2 & c.119G $>A$ & p.R40H & NA & Yes & Drug & Deceased \\
\hline 15 & LO & E2 & c. $122 \mathrm{~A}>\mathrm{C}$ & p.D41A & Inherited & Yes & Drug & Alive/yes \\
\hline 16 & LO & E4 & c.386G $>\mathrm{T}$ & p.R129C & Inherited & Yes & LT & Alive/yes \\
\hline 17 & LO & E6 & c. $562 \mathrm{G}>\mathrm{C}$ & p.G188R & Inherited & Yes & Withdraw & Withdraw \\
\hline 18 & LO & E6 & c.604C>T & p.H2O2Y & Inherited & No & LT & Alive/yes \\
\hline 19 & LO & E6 & c. $622 \mathrm{G}>\mathrm{A}$ & p.A208T & Inherited & No & Drug & Alive/yes \\
\hline 20 & LO & E8 & c.794G $>A$ & p.W265X & De novo & No & Drug & Deceased \\
\hline 21 & LO & E8 & c. $829 C>T$ & p.R277W & Inherited & No & LPD, drug & Alive/no \\
\hline 22 & LO & E8 & c. $829 C>T$ & p.R277W & Inherited & No & $\mathrm{LT}$ & Alive/yes \\
\hline 23 & LO & E8 & c. $829 C>T$ & P.R277W & Inherited & No & LPD, drug & Alive/no \\
\hline 24 & LO & E8 & c. $829 C>T$ & P.R277W & Inherited & Yes & Drug & Alive/yes \\
\hline 25 & LO & E8 & c. $829 C>T$ & p.R277W & Inherited & No & LPD, drug & Deceased \\
\hline 26 & LO & E8 & c. $829 C>T$ & p.R277W & Inherited & No & LPD, drug & Alive/Yes \\
\hline 27 & LO & E9 & $c .913 C>T$ & p.P305S & De novo & No & LPD, drug & Alive/yes \\
\hline 28 & LO & E9 & $c .919 A>G$ & p.K307E & Inherited & No & LPD & Alive/yes \\
\hline 29 & LO & E9 & c. $931 \mathrm{G}>\mathrm{A}$ & p.V311M & Inherited & No & LPD, drug & Alive/no \\
\hline 30 & LO & E10 & c. $1019 C>T$ & p.S340F & Inherited & No & LPD, drug & Alive/yes \\
\hline 31 & LO & E9 & c.929_931delAAG & p.309delE & De novo & No & Drug & Alive/no \\
\hline 32 & LO & E1-4 & E1-4 duplication & & Inherited & Yes & ND & Deceased \\
\hline 33 & LO & - & 7.8 Mb deletion of $\mathrm{Xp}$ & 11.4p21.2 & De novo & No & CVVH, Drug & Alive/yes \\
\hline 34 & LO & E2 & c.78- $2 A>G$ & IVS1-2A>G & Inherited & Yes & Drug & Deceased \\
\hline 35 & $\mathrm{EO}$ & 11 & $\mathrm{c} .77+1 \mathrm{G}>\mathrm{C}$ & IVS1+1G>C & Inherited & No & ND & Deceased \\
\hline 36 & $\mathrm{EO}$ & E9-10 & E9-10 deletion & & De novo & No & $\mathrm{LT}$ & Alive/yes \\
\hline 37 & LO & E1 & c. $3 \mathrm{G}>\mathrm{A}$ & p.M11 & De novo & No & LPD, drug & Alive/yes \\
\hline 38 & LO & E1 & $c .67 C>T$ & p.R23X & Inherited & No & LPD, drug & Alive/no \\
\hline 39 & LO & E2 & c.140delA & p.N47Tfs*17 & De novo & No & Drug & Deceased \\
\hline 40 & LO & E2 & c. $174 \mathrm{G}>\mathrm{A}$ & p.W58X & De novo & No & Withdraw & Withdraw \\
\hline 41 & LO & E3 & c. $275 \mathrm{G}>\mathrm{A}$ & p.R92Q & De novo & No & LPD, drug & Deceased \\
\hline 42 & LO & E4 & c. $317 \mathrm{G}>\mathrm{T}$ & p.G106V & Inherited & No & $\mathrm{LT}$ & Alive/no \\
\hline 43 & AS & E5 & $c .421 C>G$ & $\mathrm{p} . \mathrm{R} 141 \mathrm{G}$ & Inherited & Yes & Drug & Alive/no \\
\hline 44 & LO & E5 & c. $422 \mathrm{G}>\mathrm{A}$ & p.R141Q & Inherited & No & Drug & Deceased \\
\hline 45 & LO & E5 & C. $540 \mathrm{G}>\mathrm{C}$ & p.Q180H & De novo & No & LT & Alive/yes \\
\hline 46 & LO & E6 & c.552insGAAC & p.S185Efs*41 & De novo & No & Drug & Deceased \\
\hline 47 & LO & E6 & c. $583 \mathrm{G}>\mathrm{A}$ & p.G195R & Inherited & No & LT & Alive/yes \\
\hline 48 & LO & E6 & c. $583 \mathrm{G}>\mathrm{A}$ & p.G195R & Inherited & No & ND & Deceased \\
\hline
\end{tabular}


Table 3 (continued)

\begin{tabular}{|c|c|c|c|c|c|c|c|c|}
\hline NO. ${ }^{a}$ & Type & Exon/Intron & Nucleotide change & Amino acid change & Nature of mutation & Family history & Therapy received ${ }^{\mathbf{b}}$ & $\begin{array}{l}\text { Outcome/ } \\
\text { neurologic } \\
\text { damage }\end{array}$ \\
\hline 49 & LO & E6 & c. $626 C>T$ & p.A209V & Inherited & No & $L P D$, drug & Alive/yes \\
\hline 50 & LO & E6 & c. $626 C>T$ & p.A209V & De novo & No & Drug & Alive/yes \\
\hline 51 & LO & E7 & c. $704 A>C$ & p.Q235P & Inherited & No & LT & Alive/yes \\
\hline 52 & LO & E7 & c.703C>T & p.Q235X & Inherited & Yes & CVVH, LPD, drug & Alive/yes \\
\hline 53 & LO & E8 & c.779T>C & p.L260S & Inherited & No & ND & Deceased \\
\hline 54 & LO & E9 & c.914C>G & p.P305R & De novo & No & LT & Alive/yes \\
\hline 55 & LO & E9 & c. $944 \mathrm{~T}>\mathrm{G}$ & p.V315G & De novo & No & LT & Alive/no \\
\hline 56 & LO & 13 & c. $298+2 T>G$ & IVS3+2T>G & Inherited & No & $\mathrm{LT}$ & Alive/yes \\
\hline 57 & LO & 13 & c. $298+5 G>C$ & IVS3+5G>C & De novo & No & LPD, drug & Alive/yes \\
\hline 58 & LO & 15 & c. $540+265 G>A$ & IVS5+265G>A & De novo & No & LPD, drug & Alive/yes \\
\hline 59 & LO & 16 & c.664-1G>A & IVS6-1G>A & De novo & No & Drug & Alive/no \\
\hline 60 & LO & 17 & c. $718-1 \mathrm{G}>\mathrm{A}$ & IVS7-1G>A & De novo & No & Drug & Alive/yes \\
\hline 61 & LO & 18 & c.868-1G>C & IVS8-1G>C & De novo & No & Drug & Alive/yes \\
\hline 62 & LO & E2 & c.124_126delCTT & p.42delL & De novo & No & $\mathrm{LT}$ & Alive/no \\
\hline 63 & LO & $E 2-3$ & c.207-226del20 & & De novo & No & LPD, drug & Alive/no \\
\hline 64 & LO & E1-4 & E1-4 deletion & & Inherited & Yes & LPD, drug & Alive/yes \\
\hline 65 & LO & $E 2-4$ & E2-4 deletion & & De novo & No & Withdraw & Withdraw \\
\hline 66 & LO & E5-8 & E5-8 deletion & & De novo & No & LPD, drug & Alive/no \\
\hline 67 & LO & ND & $N D$ & ND & ND & Yes & LPD, drug & Deceased \\
\hline 68 & LO & ND & ND & ND & ND & No & Withdraw & Withdraw \\
\hline 69 & LO & ND & ND & ND & ND & No & $\mathrm{LT}$ & Alive/yes \\
\hline
\end{tabular}

EO, early onset; LO, late onset; E, exon, I, intron; LPD, low-protien diet; MV, mechanical ventilation; TPN, total parenteral nutrition; PD, peritoneal dialysis; LT, Liver transplantation; CVVH, continuous veno venous hemodiafltration; NA, not analysed; ND, not detected

a Patients 1-34 are males, and patients 35-69 are females

b Drug: Referred to L-arginine, L-Citrulline, sodium benzoate, and sodium phenylbutyrate

Table 4 Prediction of the potential pathogenic effect of novel missense variants of OTC gene

\begin{tabular}{|c|c|c|c|c|c|c|}
\hline Mutation & Domain & PROVEAN $^{a}$ & PolyPhen- $2^{b}$ & SIFT $^{c}$ & $\begin{array}{l}\text { Mutation } \\
\text { taster }^{\mathrm{d}}\end{array}$ & Conservation \\
\hline D41A & $\beta$-sheet in polar domain & Deleterious & Benign & Damaging & 126 & Conserved \\
\hline Q235P & a-helix in equatorial domain & Neutral & Benign & Damaging & 76 & Conserved \\
\hline T287। & Surface of the enzyme & Deleterious & Probably damaging & Damaging & 89 & Conserved \\
\hline P305S & Ornithine binding domain & Deleterious & Probably damaging & Damaging & 74 & Conserved \\
\hline
\end{tabular}

a PROVEAN prediction: default threshold is -2.5 , that is variants with a score equal to or below -2.5 are considered "deleterious", whereas variants with a score above -2.5 are considered "neutral"

b PolyPhen-2 prediction: probably damaging with a score of 1, in contrast, possibly damaging with a score under 1

c SIFT prediction: amino acids with probabilities $<0.05$ are predicted to be deleterious, whereas variants with a score above 0.05 are considered "neutral"

$d$ Mutation taster prediction: scores range from 0.0 to 215 . The more they score, the more deleterious protein mutations

of carbamoyl phosphate domain by structure based analysis [17]. Therefore, the mutant D41A may also affect the function of this domain and is predicted to be damaging. Q235 is located at the $\alpha$-helix in the equatorial domain [18]. The mutant P235 may affect the folding of the equatorial domain and is predicted to be damaging by SIFT and Mutation Taster, but benign by PROVEAN and PolyPhen-2. T287 is located at the surface of the enzyme, which is close to the conserved amino acid motif Ser-Met-Gly (SMG loop) that swings towards the active site to help catalysis [19]. The mutant I287 may affect this local neighborhood structure and is predicted to be deleterious by all servers. P305 is located at the C-terminal ornithine binding domain, which contains the constant 
motif Leu-His-Cys-Leu-Pro. The nonpolar amino acid proline forms a rare cis-peptide bond that is found exclusively at this location, and largely determines the shape of the ornithine site. As such, P305R mutations would be highly damaging, as observed in a male patient with neonatal-onset, which has complete enzyme deficiency [18]. Consequently, the mutant residue S305, a polar and neutral amino acid, may affect the interaction of ornithine binding and is predicted to be pathogenic by all servers.

\section{Clinical outcomes}

During 15 years of follow-up, 24 cases (16 males, 8 females) had died, 41 cases (17 males, 24 females) survived, and 4 cases ( 1 male, 3 females) withdrawn from this study. The mortality was $48.5 \%$ (16/33) in male patients and $25.0 \%(8 / 32)$ in female patients. The median age at death was 7 days (range 2 days-56 years) in males and 3.9 years (range 1 month-29 years) in females. In the early-onset group, 12 cases died following their first hyperammonemic encephalopathy at the median age of 7 days (range 2 days-44 days), resulting in a high mortality of $80 \%(12 / 15)$. In the late-onset group, except for that 4 cases lose to follow-up, 12 patients deceased at median age of 4.4 years (range 1.4 years-56 years), with a mortality of $24.5 \%$ (12/49). Estimated survival rates of these groups were shown in Fig. 3. For the 41 survivors, they survived during the initial hyperammonemic episode through nutritional support, administration of intravenous ammonia scavengers, and intensive care. Subsequent clinical progress showed neurological impairments including developmental delay, intellectual disability, learning disorder, behavior disorders, motor disorders and epilepsy, were common $(65.8 \%, 27 / 41)$.

In this study, 13 patients ( 3 males, 10 females) received liver transplantation (LT). The median age at operation was 3 years (ranged from 19 months to 11.4 years). The overall median peak blood ammonia before LT was $300 \mu \mathrm{mol} / \mathrm{L}$ (range 216-1300) in 13 patients. Prior to transplantation, the median number of hospital admissions for hyperammonemic crises was 3 (range 1-12). At an average follow-up period of 1.25 years (range $0-2.7$ years) following LT, the children could have a protein-unrestricted diet, with the blood ammonia being maintained in the normal range and no hyperammonemia episodes happened. Postoperative complications occurred in 2 patients, one (P18) having hepatapostema and the other (P36) having biliary fistula. Three patients (P42, P55, and P62) had good prognosis and underwent normal development. For the other 10 patients, neurological impairments were found before transplantation, and remained stable condition in 8 of them (P18, P22, P36, P45, P47, P51, P54, and P56) and got improved in 2 cases (P16, P69) after transplantation.

\section{Discussion}

In this study, $38.2 \%$ of males and $5.7 \%$ females had earlyonset presentation, $61.8 \%$ males and $91.4 \%$ of females displayed late-onset phenotype, which are similar to the characteristics reported in other studies [5]. However, we noticed that the number of males and females is almost same in our patients, which is inconsistent with the characteristic of this disease. The most possible reason is that a fraction of the male infants die undiagnosed in the neonatal period. In addition, high mortality was observed in our cohort as $80 \%$ in early-onset patients, $24.5 \%$ in the late-onset patients, respectively. Therefore, prompt diagnosis and emergency treatment are very important to reduce the high fatality rate. The diagnosis of OTCD is based on clinical symptoms, specific biochemical detection, and genetic testing. Since the clinical manifestations of this disease are non-specific, patients with unexplained vomiting and neurological symptoms need to be tested for blood ammonia levels. For patients with high blood ammonia, the levels of blood amino acids and urinary organic acid should be immediately tested. Trace amounts of serum citrulline and increased levels of urine orotic acid and uracil highly suggest the diagnosis of OTCD. However, normal citrulline levels have been detected in nearly three quarters (11/15) of our patients at neonatal screening and in approximately half of them at first onset, suggesting that identification of individuals with OTCD is unreliable by newborn screening program using citrulline as a biomarker, especially for late-onset or asymptomatic individuals. The amino acid profile by MS/MS cannot detect OTCD consistently because the monitoring of low citrulline levels lacks sensitivity and specificity $[20,21]$. This is why only two UCDs (argininosuccinic synthetase and lyase deficiency) were implemented in national newborn screening panels in US and some European countries (Austria, Iceland, Portugal, Hungary and Spain) [22]. In contrast, false negatives of urinary orotic acid level was low but may occurs, as shown in some of our late-onset patients (P28, P34, P69), suggesting that multiple repeat tests are required in these highly suspicious subjects. Therefore, genetic tests should be performed for confirmed diagnosis in high-risk patient as early as possible.

The recurrent sequence variants were R277W, G195R and $\mathrm{A} 209 \mathrm{~V}$ in this study. If combining with the mutations reported by Shao et al. [23], the most frequent OTC mutations in Chinese population were R277W (8/90, 8.9\%), R129H (4/90, 4.4\%), G195R (3/90, 3.3\%), A209V(2/90, 2.2\%), P225L (2/90, 2.2\%), and N61S (2/90, $2.2 \%)$. Herein we tried to discuss the genotype-phenotype correlations of these common mutations. R277W mutation arises in $\mathrm{CpG}$ dinucleotides, which recurred in discrete populations, having been reported in some male 

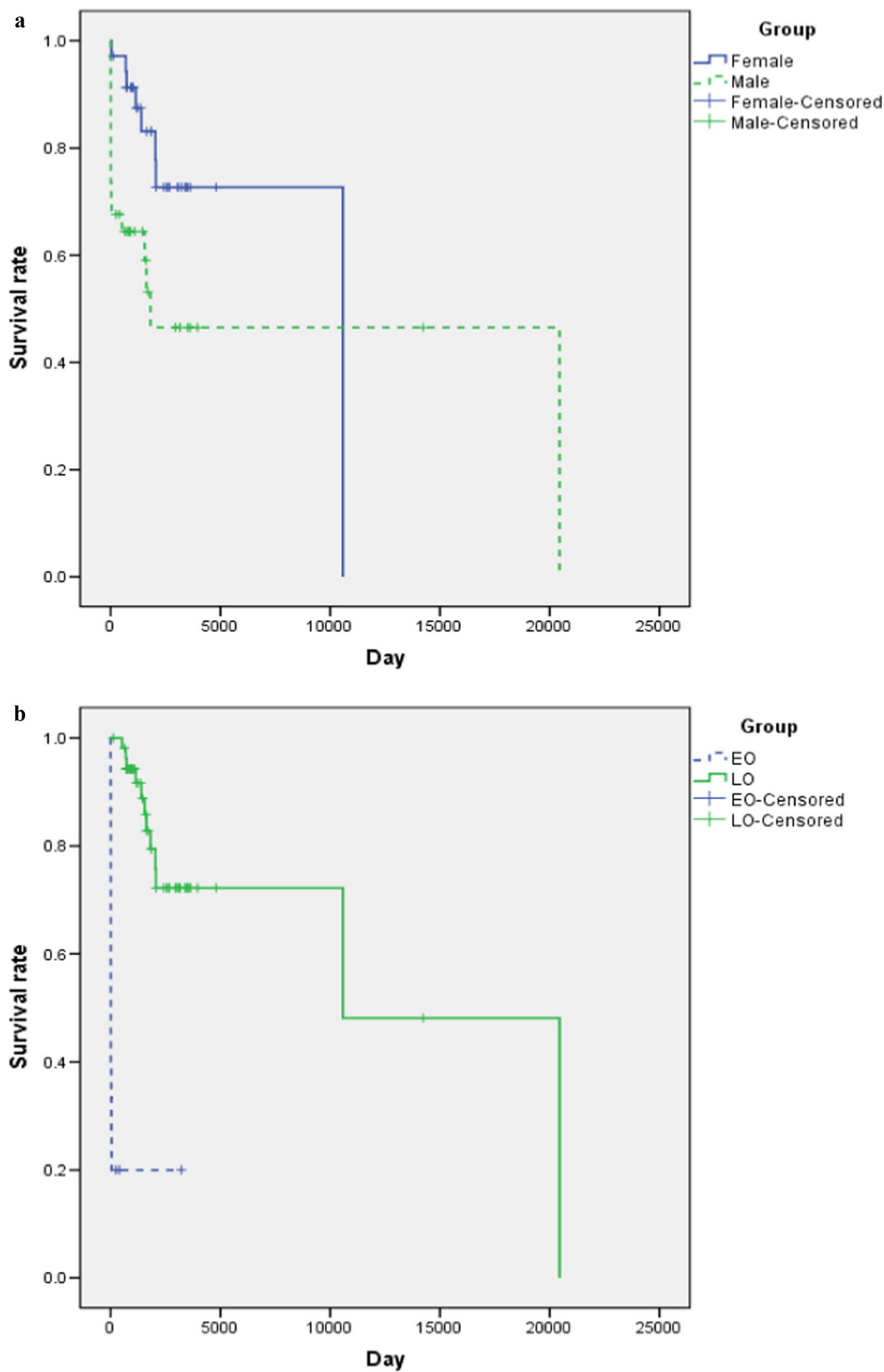

\section{Group}

$-1^{-} \cdot$ EO

$\neg$ LO

+ EO-Censored

+ LO-Censored

Fig. 3 Survival rates of different groups. a Survival rates of male and female subjects. b Survival rates of early onset and late onset subjects 
hemizygotes with asymptomatic or mild clinical manifestations [24-26]. In our study, patients with this mutant allele were all males with varied clinical symptoms. One patient (P6) experienced his first disease manifestation at 3 days old, with hyperammonemia $(700 \mu \mathrm{mol} / \mathrm{L})$ and cerebral edema, followed by neonatal death, even though he received mechanical ventilation, peritoneal dialysis, and parenteral nutrition. Among the other 6 male patients (P21-P26) with late-onset phenotype, one (P25) died after recurrent hyperammonemia encephalopathy at the age of 17 months; two cases (P22, P26) had intellectual disability; the remaining 3 patients (P21, P23, and P24) had good clinical outcomes through a low-protein diet and drug therapy. So, we expand the phenotype of this mutation, which can cause severe outcomes in males. The difference in phenotype with the same mutant allele may partly explain why the phenotype of patients depends not only on allelic heterogeneity but also on environmental factors, including daily protein intake [27]. The G195R mutation, affects a CpG dinucleotide [28], has been found in a male patient (P4) with early onset and 2 females (P47, $\mathrm{P} 48$ ) with late onset. $\mathrm{P} 4$ experienced a severe hyperammonemic episode and died a week after birth. His elder male sibling also died due to a hyperammonemia coma at 3 days following birth. P47 underwent the first hyperammonemic episode at 6 months old and experienced three instances of hyperammonemia events due to poor control of metabolic indicators via drug treatments and diet. The brain MRI showed cerebral atrophy and decreased subcortical white matter. After LT, she had good control of metabolic indexes, but little improvement in cognitive function. P48 experienced hyperammonemia during pregnancy at 29 years old, which manifested as vomiting, acro-anesthesia, limb numbness, followed by coma and rapid death. Based on the three cases described above, we suggest that this mutation could be associated with severe clinical outcomes. However, in the Spanish study, this was the third frequent mutation and had been identified in two asymptomatic female patients and one symptomatic female with late-onset phenotype [5]. As such, this indicates that the clinical outcome of this mutation may be related more to the severity at onset rather than the mutation itself. The A209V mutation occurs at a CpG site and has been reported in the Spanish population. There, one female suffered several episodes of coma from 11 to 17 months of age and remained intellectual impairment [29], while the other was a male with a late-onset phenotype, who also presented neurological damage [5]. The A209V mutation was present in two of our cases (P49, P50), both females with a late-onset phenotype. P49 was referred to us for growth delay at 18 months. Upon diagnosis of OTCD, she was prescribed a low-protein diet and treated with ammonia scavengers. However, she still experienced acute hyperammonemia crises throughout childhood. Brain MRI showed global cerebral atrophy. P50 also experienced late onset and exhibited mild intellectual impairment. The same position was also affected with pathogenic variant (A209E), which was identified in an adult female with rapidly progressive neuropsychological symptoms and coma [30]. Another variant (A209G) affects the same residue was observed in $8 / 92,400(0.009 \%)$ alleles from individuals of European background, including two hemizygous individuals in gnomAD database. Although in silico analysis predicts this variant is probably damaging, the pathogenicity of A209G is unclear based on the currently available information. As such, we speculate that A209V may be associated with a late-onset phenotype in symptomatic females or males and might carry a high risk of neurological impairment. The pathogenicity of substitutions at the same position may differ greatly.

Of the 4 novel missense mutations, T287I was associated with early-onset or severe clinical manifestation. The male patient (P7) had gastrointestinal symptoms at the age of 5 days and was suspected of OTCD because of family history, with two uncles and a cousin dying of this disorder at 13 and 9 years old, respectively. Currently, he has good control of his ammonia levels and normal mental development due to early diagnosis and treatment. The other 3 novel mutations were associated with the late-onset phenotype. The D41A mutation was found in a male patient (P15) diagnosed by neonatal screening. He received conventional therapy, but metabolic characteristics were not in good control. He suffered the first onset at 1.8 years of age, and presented developmental delay and intellectual disability. His family history showed an elder male sibling died at eight months old with unclear diagnosis. The Q235P was detected in a female patient (P51). She experienced the first hyperammonemic episode at 3 years of age and suffered recurrent hyperammonemic events until accepting liver transplantation at 11.4 years of age. Poor psycho-neurodevelopment has been observed. P305S was found in a male patient (P27) with severe encephalatrophy. He had frequent epileptic seizures and cannot raise his head or sit by himself at 2.2 years old.

The gross deletions and duplications account for 16.7\% of all mutations in this study. As 3 patients with undetected variants did not perform MPLA screening, the relative frequency of these mutations may be biased and thus somewhat underestimated. Therefore, MPLA analysis is necessary for all cases in which no mutation is detected by direct sequencing. Among the 9 patients with such mutations, 4 male subjects had bad clinical outcomes. Two of them (P9, P10) died from the first acute hyperammonemia encephalopathy episode in a 
week after birth. P32 died at 5 years old due to serious hyperammonemia (NH3, $2500 \mu \mathrm{mol} / \mathrm{L})$ and had a family history of OTCD, with two uncles with similar symptoms who died during childhood. P33 survived the first hyperammonemic episode owing to urgent and continuous venovenous hemodiafiltration. However, he had severe psychomotor delays. He walks unsteadily and has poor language skills at the age of 4 years. In the 5 female cases, one (P36) displayed a neonatal phenotype and suffered recurrent hyperammonemia episodes. She received a liver transplant at 7.5 years of age and then the hyperammonemic episodes stopped. However, the impairments in cognitive function persisted, with poor performance and failing grade in grade two. Among the remaining 4 female cases (P63-P66) with late onset, 2 of them now have good control of their metabolic indexes and normal mental development; one had mild intellectual impairment and the other is lost to follow up. Therefore, we consider that gross deletion or duplication of OTC gene in males can cause severe clinical outcomes, while in females, the severity depends on the onset age and other factors (X-inactivation pattern or environment).

LT has emerged as a definitive treatment for the risk of metabolic decompensation in urea cycle disorders, including OTCD, with excellent post-transplant survival rates and good metabolic outcomes [31]. LT should be considered early in neonatal cases and those considered refractory to optimal medical therapy. In a study by $\mathrm{Yu}$ et al. [32], of the 136 patients who underwent LT for OTCD, the majority were males. However, the majority (76.9\%) of our patients who underwent LT are females. This can probably be attributed to two main factors: (1) Male patients have higher mortality of $48.5 \%$ and one third of males have neonatal death following the first hyperammonemic episode. (2) Since females have higher survival rate, some of them suffered from a high frequency of hyperammonemic crises, which urged them to receive LT. It have been reported that neurologic outcomes can be improved by LT before the age of one [33], and the effects on cognition can be stabilized to premorbid function in the long term [34]. However, none of our patients received LT before 1 year of age, with the youngest pediatric LT being performed in a girl of 1 year and 7 months old. This is likely due to the shortage in the selection of the timing and indication for LT by clinicians, rather than donor shortage, economic pressures and technical reasons, because children under 1 year of age are the main $(61 \%)$ recipients of pediatric LT in China after 2012, with one-year and five-year survival rates of 90.5\% and $83.6 \%$, respectively (data from China Liver Transplant Registry). In addition, the five-year survival rate and long-term neurologic outcomes of our patients remain unknown, which require longer follow-up.
In conclusion, we described clinical symptoms, biochemical features, clinical outcomes, and molecular analysis of 69 OTCD patients. There is an equal sex ratio in our patients. The mortality rate is extremely high among early-onset patients. We consider that the evaluation of serum citrulline level is of limited value in diagnosis of OTCD, while urine orotic acid detection and genetic testing are more helpful. A total of 53 different mutations have been identified and 18 of them are novel.

\section{Supplementary information}

Supplementary information accompanies this paper at https://doi. org/10.1186/s13023-020-01606-2.

Additional file 1: Table 1S. Primers and annealing temperature for PCR amplification of OTC-specific fragments.

\section{Abbreviations}

F: Female; M: Male; d: Days; m: Months; y: Years; EO: Early onset; LO: Late onset; AS: Asymptomatic; CIT: Blood citrulline; GLN: Blood glutamine; AMON: Blood ammonia; ALT: Alanine aminotransferase; E: Exon; I: Intron; LPD: Lowprotien diet; MV: Mechanical ventilation; TPN: Total parenteral nutrition; PD: Peritoneal dialysis; LT: Liver transplantation; CVVH: Continuous veno venous hemodiafltration.

\section{Acknowledgements}

We are grateful to the patients and their families for their participation in this study. We also express our gratitude to all of the pediatricians who provided patient data and blood samples.

\section{Authors' contributions}

Deyun Lu, Feng Han and Lianshu Han conceived and designed the study. Yu Wang, Wenjun Ji and Xia Zhan did laboratory work (MS/MS, GC/MS, PCR and sequencing). Feng Han collected the clinical data. Deyun Lu did data analysis and wrote the draft. Lianshu Han revised the manuscript and acted as the guarantor of the study. Xuefan Gu, Wenjuan Qiu, Huiwen Zhang, Jun Ye and Lili Liang reviewed critically the article. All authors read and approved the final manuscript.

\section{Funding}

This study was supported by the National Key Research and Development Program of China (Grant Number: 2016YFC0901505) and Precision Medical Research of National Key Research and Development Program (2016YFC0905100 to WJQ and XFG).

\section{Availability of data and materials}

The datasets used during and/or analyzed during the current study are available from the corresponding author on request.

\section{Ethics approval and consent to participate}

The study was approved by the ethical committee of Xin Hua Hospital affiliated to Shanghai Jiao Tong University School of Medicine. Written informed consent was obtained from all recruited subjects or their legal guardians (Approval No. XHEC-D-2020-025).

\section{Consent for publication}

All authors agreed on the manuscript.

\section{Competing interest}

The authors declare that they have no conflict of interest.

\section{Author details}

${ }^{1}$ Department of Pediatric Endocrinology and Genetic Metabolism, Shanghai Institute for Pediatric Research, Xinhua Hospital Affiliated To Shanghai Jiao Tong University School of Medicine, Shanghai, China. ${ }^{2}$ Department 
of Neurology, Shanghai Children's Medical Center Affiliated to Shanghai Jiao Tong University School of Medicine, National Children's Medical Center, Shanghai, China.

Received: 17 May 2020 Accepted: 3 November 2020 Published online: 03 December 2020

\section{References}

1. Caldovic L, Abdikarim I, Narain S, Tuchman M, Morizono H. Genotypephenotype correlations in ornithine transcarbamylase deficiency: a mutation update. J Genet Genomics. 2015:42:181-94.

2. Pizzi MA, Alejos D, Hasan TF, Atwal PS, Krishnaiengar SR, Freeman WD. Adult presentation of ornithine transcarbamylase deficiency: 2 illustrative cases of phenotypic variability and literature review. Neurohospitalist. 2019:9:30-6.

3. Horwich AL, Fenton WA, Williams KR, Kalousek F, Kraus JP, Doolittle RF, et al. Structure and expression of a complementary DNA for the nuclear coded precursor of human mitochondrial ornithine transcarbamylase. Science. 1984;224:1068-74.

4. Matsuda I, Tanase S. The Ornithine Transcarbamylase (OTC) Gene: mutations in 50 Japanese families with OTC deficiency. Am J Med Genet. 1997:71:378-83.

5. Martín-Hernández E, Aldámiz-Echevarría L, Castejón-Ponce E, PedrónGiner C, Couce ML, Serrano-Nieto J, et al. Urea cycle disorders in Spain: an observational, cross-sectional and multicentric study of 104 cases. Orphanet J Rare Dis. 2014;9:187.

6. Tuchman M, Plante RJ, Garcia-Perez MA, Rubio V. Relative frequency of mutations causing ornithine transcarbamylase deficiency in 78 families. Hum Genet. 1996:97:274-6.

7. Musalkova D, Sticova E, Reboun M, Sokolova J, Krijt J, Honzikova J, et al. Variable X-chromosome inactivation and enlargement of pericentral glutamine synthetase zones in the liver of heterozygous females with OTC deficiency. Virchows Arch. 2018;472:1029-39.

8. Chongsrisawat V, Damrongphol P, Ittiwut C, Ittiwut R, Suphapeetiporn $K$, Shotelersuk $V$. The phenotypic and mutational spectrum of Thai female patients with ornithine transcarbamylase deficiency. Gene. 2018;679:377-81

9. Yang N, Han LS, Ye J, Qiu WJ, Zhang HW, Gao XL, et al. Diseases spectrum of metabolism disease of amino acid, organic acid and fatty acid oxidation in neonates. J Clin Pediatr. 2012;30:805-8.

10. Brassier A, Gobin S, Arnoux JB, Valayannopoulos V, Habarou F, Kossorotoff $\mathrm{M}$, et al. Long-term outcomes in Ornithine Transcarbamylase deficiency: a series of 90 patients. Orphanet J Rare Dis. 2015;10:58.

11. Summar ML, Dobbelaere D, Brusilow S, Lee B. Diagnosis, symptoms, frequency and mortality of 260 patients with urea cycle disorders from a 21-year, multicentre study of acute hyperammonaemic episodes. Acta Paediatr. 2008;97:1420-5.

12. Han LS, Ye J, Qiu WJ, Gao XL, Wang Y, Gu XF. Selective screening for inborn errors of metabolism on clinical patients using tandem mass spectrometry in china: a four-year report. J Inherit Metab Dis. 2007;30:507-14

13. Han LS, Ye J, Qiu WJ, Gao XL, Wang Y, Jin J, et al. Diagnosis of inborn errors of metabolism using tandem mass spectrometry and gas chromatography mass spectrometry. Zhonghua Yi Xue Za Zhi. 2008;88:2122-6.

14. Sun Y, Hu G, Liu H, Zhang X, Huang Z, Yan H, et al. Further delineation of the phenotype of truncating KMT2A mutations: the extended Wiedemann-Steiner syndrome. Am J Med Genet A. 2017;173:510-4.

15. Gong ZW, Han LS, Ye J, Qiu WJ, Zhang HW, Yu YG, et al. Applying multiplex ligation-dependent probe amplification in the diagnosis of 5 cases with ornithine transcarbamylase deficiency. Chin J Pediatr. 2016;54:437-40.

16. Quental R, Azevedo L, Rubio V, Diogo L, Amorim A. Molecular mechanisms underlying large genomic deletions in ornithine transcarbamylase (OTC) gene. Clin Genet. 2009;75:457-64.

17. Arranz JA, Riudor E, Marco-Marín C, Rubio V. Estimation of the total number of disease-causing mutations in ornithine transcarbamylase (OTC) deficiency. Value of the OTC structure in predicting a mutation pathogenic potential. J Inherit Metab Dis. 2007;30:217-26.
18. Tuchman M, Morizono H, Reish O, Yuan X, Allewell NM. The molecular basis of ornithine transcarbamylase deficiency: modelling the human enzyme and the effects of mutations. J Med Genet. 1995;32:680-8.

19. Yamaguchi S, Brailey LL, Morizono H, Bale AE, Tuchman M. Mutations and polymorphisms in the human ornithine transcarbamylase (OTC) gene. Hum Mutat. 2006;27:626-32.

20. Cavicchi C, Malvagia S, la Marca G, Gasperini S, Donati MA, Zammarchi E, et al. Hypocitrullinemia in expanded newborn screening by LC-MS/MS is not a reliable marker for ornithine transcarbamylase deficiency. J Pharm Biomed Anal. 2009;49:1292-5.

21. Loeber JG, Burgard P, Cornel MC, Rigter T, Weinreich SS, Rupp K, et al. Newborn screening programmes in Europe; arguments and efforts regarding harmonization. Part 1. From blood spot to screening result. J Inherit Metab Dis. 2012;35:603-11.

22. Summar ML, Koelker S, Freedenberg D, Le Mons C, Haberle J, Lee $\mathrm{HS}$, et al. The incidence of urea cycle disorders. Mol Genet Metab. 2013;110:179-80.

23. Shao $Y$, Jiang $M$, Lin $Y$, Mei $H$, Zhang W, Cai Y, et al. Clinical and mutation analysis of 24 Chinese patients with ornithine transcarbamylase deficiency. Clin Genet. 2017:92:318-22.

24. Hata A, Matsuura T, Setoyama C, Shimada K, Yokoi T, Akaboshi I, et al. A novel missense mutation in exon 8 of the ornithine transcarbamylase gene in two unrelated male patients with mild ornithine transcarbamylase deficiency. Hum Genet. 1991;87:28-32.

25. Matsuura T, Hoshide R, Setoyama C, Shimada K, Hase Y, Yanagawa T, et al. Four novel gene mutations in five Japanese male patients with neonatal or late onset OTC deficiency: application of PCR-single-strand conformation polymorphisms for all exons and adjacent introns. Hum Genet. 1993:92:49-56.

26. Storkanova G, Vlaskova H, Chuzhanova N, Zeman J, Stranecky V, Majer F, et al. Ornithine carbamoyltransferase deficiency: molecular characterization of 29 families. Clin Genet. 2013;84:552-9.

27. Matsuda I, Matsuura T, Nishiyori A, Komaki S, Hoshide R, Matsumoto T, et al. Phenotype variability in male patients carrying the mutant ornithine transcarbamylase (OTC) allele, Arg40His, ranging from a child with an unfavourable prognosis to an asymptomatic older adult. J Med Genet. 1996;33:645-8.

28. Tuchman M, Plante RJ. Mutations and polymorphisms in the human ornithine transcarbamylase gene: mutation update addendum. Hum Mutat. 1995:5:293-5.

29. García-Pérez MA, Paz Briones PS, García-Munñoz MJ, Rubio V. A splicing mutation, a nonsense mutation (Y167X) and two missense mutations (1159T and A209V) in Spanish patients with ornithine transcarbamylase deficiency. Hum Genet. 1995;96:549-51.

30. Bailly P, Noury JB, Timsit S, Ben SD. Teaching Neurolmages: Ornithine transcarbamylase deficiency revealed by a coma in a pregnant woman. Neurology. 2015;85:e146-7.

31. Wilnai Y, Blumenfeld YJ, Cusmano K, Hintz SR, Alcorn D, Benitz WE, et al. Prenatal treatment of ornithine transcarbamylase deficiency. Mol Genet Metab. 2018;123:297-300

32. Yu L, Rayhill SC, Hsu EK, Landis CS. Liver transplantation for urea cycle disorders: analysis of the united network for organ sharing database. Transplant Proc. 2015:47:2413-8.

33. Campeau PM, Pivalizza PJ, Miller G, McBride K, Karpen S, Goss J, et al. Early orthotopic liver transplantation in urea cycle defects: follow up of a developmental outcome study. Mol Genet Metab. 2010;100(Suppl 1):S84-7.

34. Crowe L, Anderson V, Hardikar W, Boneh A. Cognitive and behavioural outcomes of paediatric liver transplantation for Ornithine Transcarbamylase Deficiency. JIMD Rep. 2019:43:19-25.

\section{Publisher's Note}

Springer Nature remains neutral with regard to jurisdictional claims in published maps and institutional affiliations. 